\title{
Prognostic significance of malnutrition for long-term mortality in community-acquired pneumonia: a propensity score matched analysis
}

\author{
Hye Ju Yeo ${ }^{1,2,}$, Ki Sup Byun ${ }^{1,2,}$, Junhee $\mathrm{Han}^{3}$, June Hyun $\mathrm{Kim}^{4}$, Seung Eun Lee ${ }^{1,2}$, Seong Hoon Yoon ${ }^{1,2}$,
} Doosoo Jeon ${ }^{1,2}$, Yun Seong Kim ${ }^{1,2}$, and Woo Hyun $\mathrm{Cho}^{1,2}$

\begin{abstract}
${ }^{1}$ Department of Pulmonology and Critical Care Medicine, ${ }^{2}$ Research Institute for Convergence of Biomedical Science and Technology, Pusan National University Yangsan Hospital, Yangsan; ${ }^{3}$ Department of Statistics, Hallym University, Chuncheon; ${ }^{4}$ Department of Internal Medicine, Pusan National University Yangsan Hospital, Yangsan, Korea
\end{abstract}

Received: January 25, 2018 Revised : April 21, 2018 Accepted: June 13, 2018

\section{Correspondence to}

Woo Hyun Cho, M.D.

Department of Pulmonology and Critical Care Medicine, Research Institute for Convergence of Biomedical Science and Technology, Pusan National University Yangsan Hospital, 20 Geumo-ro, Mulgeum-eup,

Yangsan 50612, Korea

Tel: $+82-55-360-2120$

Fax: +82-55-360-2157

E-mail: popeyeso212@hanmail.net

*These authors contributed equally to this work.
Background/Aims: The impact of malnutrition on the outcome of hospitalized adults with community-acquired pneumonia (CAP) has not been fully investigated. This study evaluated the prevalence and prognostic significance of malnutrition in a Korean population with CAP.

Methods: In total, 198 patients with CAP from November 2014 to September 2015 were analyzed retrospectively. We assessed the prevalence of malnutrition and the risk factors for 2-year mortality. Furthermore, we divided the patients into two groups: elderly (age $\geq 65$ years, $\mathrm{n}=131$ ) and non-elderly (age $<65$ years, $\mathrm{n}=67$ ). Subgroup analyses were performed in the elderly group through propensity score matching.

Results: The prevalence of malnutrition was $39.4 \%$, and the proportion of patients with malnutrition was significantly higher ( $53.4 \%$ vs. $11.9 \%, p<0.001)$ in the elderly group than in the non-elderly group. In-hospital mortality, 1-year mortality, and 2-year mortality rates were $4.5 \%, 19.2 \%$, and $26.8 \%$, respectively. Multivariate Cox regression analyses revealed that malnutrition (odds ratio [OR], 2.52; $95 \%$ confidence interval [CI], 1.39 to $4.60 ; p=0.002$ ) and the Charlson comorbidity index score (OR, 1.30; 95\% CI, 1.17 to 1.45; $\mathrm{p}<0.001$ ) were associated with 2-year mortality.

Conclusions: Malnutrition was common and associated with a poor long-term outcome in patients with CAP, particularly the elderly. A routine nutritional assessment at admission is mandatory as a first step for appropriate nutritional therapy.

Keywords: Pneumonia; Aged; Functional status; Malnutrition; Prognosis

\section{INTRODUCTION}

Community-acquired pneumonia (CAP) is one of the main causes of morbidity and mortality, and the prognosis depends on many factors including nutritional status [1-4]. Malnutrition is associated with many ad- verse clinical outcomes including longer length of hospital stay, increased morbidity and mortality, and increased hospital costs $[5,6]$. Therefore, early recognition of malnutrition at admission and early nutritional therapy in hospitalized patients are important in addition to treating the underlying diseases. 
In particular, malnutrition in the elderly is a major health problem [7]. In Korea, the population is aging at a faster rate than in other countries, and it is expected that the numbers of elderly individuals with CAP will increase in line with the increase in the elderly population [8]. Although many Korean studies have reported the incidence of CAP and its associated risk factors, there is a lack of information regarding malnutrition [9-12]. Furthermore, no comparative information is available on the prevalence of malnutrition in these patients. We compared the prevalence of malnutrition between elderly and non-elderly groups and assessed the risk factors for long-term mortality. Furthermore, we focused on malnutrition in the elderly with CAP and performed subgroup analyses to better understand the influence of age and nutritional status.

\section{METHODS}

\section{Patients}

Eligible patients included those aged $\geq 18$ years old who were admitted to a tertiary teaching hospital (1,200 beds) due to pneumonia between November 2014 and September 2015. CAP was defined as an acute community-acquired infection of the pulmonary parenchyma and was confirmed radiologically. The inclusion criteria comprised the presence of new chest radiographic infiltrates on admission, and at least one major or two minor criteria, as follows. The major inclusion criteria were cough, sputum, or fever $\left(38^{\circ} \mathrm{C}\right)$, and the minor inclusion criteria were dyspnea, pleuritic chest pain, pulmonary consolidation, and leukocytosis $>12,000 / \mathrm{mL}$. We excluded patients who had been admitted to the hospital within the previous 90 days, immunosuppressed patients (patients with acquired immunodeficiency syndrome, or those receiving chemotherapy and/or corticosteroids [equivalent prednisone dose: $20 \mathrm{mg} /$ day]), and those who had required regular hemodialysis ( $>2$ sessions per week) within the previous 30 days. All patients who were transferred from a nursing home were excluded to rule out healthcare-associated pneumonia. Patients classified as "do not resuscitate" were excluded. The patients were divided into two groups: an elderly group (age $\geq 65$ years) and a non-elderly group (age $<65$ years). Malnutrition was assessed by clinical nutritionists within 48 hours of admission according to our hospital protocol, which was structured based on the Academy of Nutritional and Dietetics consensus criteria [13,14]. No single parameter is definitive for adult malnutrition; therefore, patients who were identified as having two or more of the following six characteristics were diagnosed with malnutrition: insufficient energy intake (duration $=$ week, amount $=\%$ of target calorie), weight loss (duration $=$ week, amount $=\%$ loss), loss of muscle mass (quadriceps and deltoids), loss of subcutaneous fat (triceps, chest), localized or generalized fluid accumulation (ankle, sacrum, and ascites), and diminished functional status measured using handgrip strength. Subsequently, malnutrition was classified into five criteria according to International Classification of Disease 9 clinical modification codes such as kwashiorkor, marasmus, protein calorie malnutrition, moderate malnutrition, or mild malnutrition. Chronic malnutrition was defined as a patient who had a reduced calorie intake $<75 \%$ for $>1$ month and/or who lost more than $5 \%$ of their body weight in more than 1 month.

\section{Data collection and analyses}

Data were collected and analyzed retrospectively. The following information was reviewed from patient medical records: demographic data, pneumonia severity index (PSI), risk factors, variables associated with nutritional status, the Charlson comorbidity index (CCI), the metabolic index, and clinical outcome. The survival of all patients was confirmed at each time point by confirming the loss of health insurance qualification.

\section{Statistical analyses}

Statistical analyses were conducted using R software version 3.0.1 (http://www.R-project.org). Descriptive statistics included frequency analyses (percentages) for categorical variables and means, and standard deviations for continuous variables. To test for differences in clinical and laboratory findings according to age group or nutrition group, the Wilcoxon rank-sum test was used for continuous variables, as appropriate. The chi-square test or Fisher's exact test was used for discrete variables. Cox regression analyses were performed to identify predictors of 2-year mortality. After univariate analyses, significant and borderline $(p \leq 0.05)$ values were entered into stepwise backward multivariate Cox regression analyses. Two-way interaction terms were tested among the re- 
maining significant variables. To approximate a random assignment in the elderly, the propensity score matching method was used to create sets of patient groups with and without malnutrition specifically, which were adjusted for their ages and rates of intensive care unit (ICU) admission [15]. We used a genetic matching algorithm that automates the process of searching for a good match result $[16,17]$. A genetic search algorithm can find a set of weights for each covariate such that the optimal balance can be achieved after matching. A genetic matching method was used to obtain the optimal ratio between the two groups without the ratio being limited to 1:1 or 1:2. The process was repeated until we obtained datasets with no statistically significant differences for age and rates of ICU admission. A two-sided $p<0.05$ was considered statistically significant.

\section{Ethics statement}

This study was approved by the Institutional Review Board of Pusan National University Yangsan Hospital (05-2017-180). The requirement of informed consent from the patients was waived due to the retrospective nature of this study.

\section{RESULTS}

In total, 478 patients admitted to our hospital due to pneumonia from November 2014 to September 2015 were retrospectively reviewed. Of these patients, 198 were included in this analysis (Fig. 1). The patients were divided into two groups: elderly (age $\geq 65$ years, $\mathrm{n}=131$ ) and non-elderly (age $<65$ years, $\mathrm{n}=67$ ).

\section{Clinical characteristics of the elderly and non-elder- ly groups}

The characteristics of the patients according to age group are shown in Table 1 . The overall prevalence of malnutrition was $39.4 \%$. The proportion of patients with malnutrition was significantly higher (53.4\% vs. $11.9 \%$, p $<0.001$ ) and body mass index (BMI) was significantly lower $\left(21.1 \pm 3.5 \mathrm{~kg} / \mathrm{m}^{2}\right.$ vs. $\left.22.7 \pm 4.0 \mathrm{~kg} / \mathrm{m}^{2}, p=0.004\right)$ in the elderly group than in the non-elderly group. In particular, the proportions of patients with kwashiorkor (9.2\% vs. $0 \%, p=0.011)$, protein calorie malnutrition $(16.8 \%$ vs. $1.5 \%, p=0.001$, and malnutrition of a mild degree

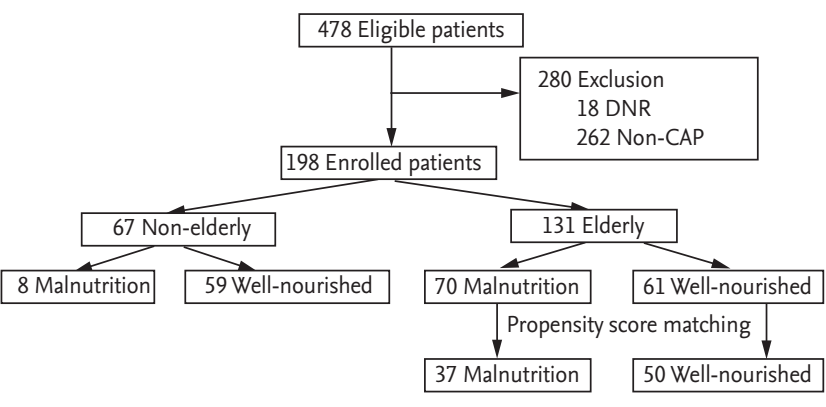

Figure 1. Enrollment diagram. About 39\% of the hospitalized patients with community-acquired pneumonia had malnutrition at admission. Approximately one half of the elderly patients with community-acquired pneumonia (CAP) were initially malnourished. DNR, do not resuscitate.

(22.1\% vs. $10.4 \%, p=0.044$ ) were significantly higher in the elderly group than in the non-elderly group. The ratio of chronic malnutrition was also significantly higher $(24.4 \%$ vs. $3.0 \%, p<0.001)$ in the elderly group than in the non-elderly group. No significant difference in the metabolic index for nutrition was observed between the two groups. The CCI score was significantly higher $(5.7 \pm 2.1$ vs. $2.5 \pm 1.9, p<0.001)$, and the PSI score was significantly lower $(82.7 \pm 33.2$ vs. $90.3 \pm 24.2, p<0.001)$ in the elderly group than in the non-elderly group. The proportion of patients with a PSI classification above IV was lower in the elderly group than in the non-elderly group (PSI class IV and V: $39 \%$ vs. $44.8 \%, p=0.039$ ).

\section{Clinical outcomes of the elderly and non-elderly groups}

The discharge rate (81.7\% vs. 97.0, $p=0.005)$, intermediate survival rate (1-year survival: $75.6 \%$ vs. $91.0 \%, p=$ 0.009 ), and long-term survival rate (2-year survival: $67.2 \%$ vs. $85.1 \%, p=0.007$ ) were significantly lower in the elderly group than in the non-elderly group (Table 2 ).

\section{Risk factors for 2-year mortality}

Age, nutritional status, PSI, and CCI were evaluated to identify independent risk factors for 2-year mortality. Univariate analyses showed that elderly ( $\geq 65$ years) (odds ratio [OR], 2.70; 95\% confidence interval [CI], 1.26 to 5.38; $p=0.005$ ), malnutrition (OR, 3.82; 95\% CI, 2.16 to 6.76; $p<$ 0.001 ), CCI score (OR, 1.37; 95\% CI, 1.24 to 1.52; $p<0.001$ ), renal replacement therapy (OR, 3.87; 95\% CI, 1.64 to 9.13; $p=0.002$ ), and BMI (OR, 0.89; $95 \% \mathrm{CI}, 0.82$ to $0.98 ; p=$ 
Table 1. Baseline characteristics of study population according to age group

\begin{tabular}{|c|c|c|c|}
\hline Variable & Elderly $(\mathrm{n}=131)$ & Non-elderly $(n=67)$ & $p$ value \\
\hline Age, yr & $75 \cdot 5 \pm 6.5$ & $50.9 \pm 11.1$ & $<0.001$ \\
\hline Male sex & $74(56.5)$ & $42(62.7)$ & 0.402 \\
\hline $\mathrm{BMI}, \mathrm{kg} / \mathrm{m}^{2}$ & $21.1 \pm 3.5$ & $22.7 \pm 4.0$ & 0.004 \\
\hline Malnutrition & $70(53.4)$ & $8(11.9)$ & $<0.001$ \\
\hline \multicolumn{4}{|l|}{ Classification of malnutrition } \\
\hline Kwashiorkor & $12(9.2)$ & o & 0.011 \\
\hline Marasmus & $6(4.6)$ & $\mathrm{O}$ & 0.075 \\
\hline Protein-Calorie malnutrition & $22(16.8)$ & $1(1.5)$ & 0.001 \\
\hline Malnutrition of moderate degree & $2(1.5)$ & $\mathrm{O}$ & 0.309 \\
\hline Malnutrition of mild degree & $29(22.1)$ & $7(10.4)$ & 0.044 \\
\hline Chronic malnutrition & $32(24 \cdot 4)$ & $2(3.0)$ & $<0.001$ \\
\hline Charlson comorbidity index & $5 \cdot 7 \pm 2.1$ & $2.5 \pm 1.9$ & $<0.001$ \\
\hline \multicolumn{4}{|l|}{ Metabolic index for nutrition } \\
\hline Albumin & $3.5 \pm 0.6$ & $3.6 \pm 0.6$ & 0.380 \\
\hline Total protein & $6.7 \pm 0.8$ & $6.6 \pm 0.8$ & 0.541 \\
\hline CRP & $12.7 \pm 9.0$ & $15 \cdot 3 \pm 10.3$ & 0.067 \\
\hline Glucose & $159 \cdot 3 \pm 119.1$ & $155 \cdot 7 \pm 75 \cdot 5$ & 0.827 \\
\hline Hemoglobin & $12.1 \pm 2.1$ & $12.7 \pm 2.2$ & 0.073 \\
\hline Hematocrit & $35 \cdot 4 \pm 5 \cdot 7$ & $36.6 \pm 5.9$ & 0.157 \\
\hline PSI score & $82.7 \pm 33.2$ & $90.3 \pm 24.2$ & $<0.001$ \\
\hline PSI class & & & 0.039 \\
\hline I, II & $53(40.5)$ & $16(23.9)$ & \\
\hline III & $27(20.6)$ & $21(31.3)$ & \\
\hline IV & $39(29.8)$ & $27(40.3)$ & \\
\hline $\mathrm{V}$ & $12(9.2)$ & $3(4 \cdot 5)$ & \\
\hline
\end{tabular}

Values are presented as mean $\pm \mathrm{SD}$ or number (\%).

BMI, body mass index; CRP, C-reactive protein; PSI, pneumonia severity index.

Table 2. Clinical outcomes of the total population

\begin{tabular}{|c|c|c|c|}
\hline Variable & Elderly $(\mathrm{n}=131)$ & Non-elderly $(n=67)$ & $p$ value \\
\hline \multicolumn{4}{|l|}{ Clinical outcome } \\
\hline ICU admission & $11(8.4)$ & $6(9.0)$ & 0.894 \\
\hline Hospital stay, day & $16.1 \pm 15.9$ & $13.6 \pm 7.5$ & 0.220 \\
\hline Hospital mortality & $7(5 \cdot 3)$ & $2(3.0)$ & 0.451 \\
\hline Discharge to home & $107(81.7)$ & $65(97.0)$ & 0.005 \\
\hline Discharge to nursing facility & $17(13.0)$ & o & 0.005 \\
\hline Readmission within 2 yr & $17(13.0)$ & $6(9.0)$ & 0.403 \\
\hline 1-yr survival & $99(75.6)$ & $61(91.0)$ & 0.009 \\
\hline 2-yr survival & $88(67.2)$ & $57(85.1)$ & 0.007 \\
\hline
\end{tabular}

Values are presented as number (\%) or mean $\pm \mathrm{SD}$.

ICU, intensive care unit. 
Table 3. Multivariate Cox regression analysis for 1-year mortality and 2-year mortality

\begin{tabular}{|c|c|c|c|c|}
\hline \multirow{3}{*}{ Variable } & \multicolumn{4}{|c|}{ Multivariate analysis } \\
\hline & \multicolumn{2}{|c|}{ Total } & \multicolumn{2}{|c|}{ Elderly } \\
\hline & OR $(95 \% \mathrm{CI})$ & $p$ value & OR $(95 \% \mathrm{CI})$ & $p$ value \\
\hline \multicolumn{5}{|l|}{ 1-yr mortality } \\
\hline Age & & & $1.12(1.05-1.20)$ & 0.001 \\
\hline Malnutrition & $3.01(1.40-6.47)$ & 0.005 & $3.86(1.32-11.35)$ & 0.014 \\
\hline CCI score & $1.30(1.15-1.48)$ & $<0.001$ & $1.43(1.21-1.70)$ & $<0.001$ \\
\hline Renal replacement therapy & $2.80(1.12-7.01)$ & 0.028 & $3.13(1.13-8.68)$ & 0.028 \\
\hline \multicolumn{5}{|l|}{ 2-yr mortality } \\
\hline Age & & & $1.06(1.01-1.12)$ & 0.030 \\
\hline Malnutrition & $2.52(1.39-4.60)$ & 0.002 & $3.06(1.44-6.50)$ & 0.004 \\
\hline CCI score & $1.30(1.17-1.45)$ & $<0.001$ & $1.37(1.18-1.58)$ & $<0.001$ \\
\hline
\end{tabular}

OR, odds ratio; CI, confidence interval; CCI, Charlson comorbidity index.

0.012) were associated with 2-year mortality. All of these variables were included in the Cox regression analysis model. Multivariate analyses showed that malnutrition (OR, 2.52; $95 \%$ CI, 1.39 to $4.60 ; p=0.002$ ) and CCI score (OR, 1.30; $95 \%$ CI, 1.17 to $1.45 ; p<0.001$ ) were associated with 2-year mortality (Table 3).

\section{Clinical characteristics according to the presence of malnutrition within the elderly group}

The characteristics of the elderly patients according to their nutritional status are shown in Table 4. Before propensity score matching, the malnourished group was significantly older (77.2 years vs. 73.5 years, $p=0.001$ ). BMI and serum levels of total protein were lower in the malnourished group than in the well-nourished group (BMI: $19.8 \pm 3.0 \mathrm{~kg} / \mathrm{m}^{2}$ vs. $22.5 \pm 3.5 \mathrm{~kg} / \mathrm{m}^{2}, p<0.001$; serum levels of total protein: $6.6 \pm 0.8 \mathrm{~g} / \mathrm{dL}$ vs. $6.8 \pm 0.8$ $\mathrm{g} / \mathrm{dL}, \mathrm{p}=0.046)$. Use of a vasopressor and mechanical ventilation rates were significantly higher in the malnourished group at ICU admission (ICU admission: $14.3 \%$ vs. $1.6 \%, p=0.009$; use of vasopressor: $11.4 \%$ vs. $1.6 \%, p=0.027$; mechanical ventilation: $12.9 \%$ vs. $1.6 \%$, $p=0.016)$. We performed propensity score matching analyses in the elderly group to reduce the effects of potential confounders, such as age and disease severity.

Propensity score matching analyses between malnourished and well-nourished patients in the elderly group

After propensity score matching, the final analyses in- cluded 87 patients, composed of a malnourished group $(\mathrm{n}=37)$ and a well-nourished group $(\mathrm{n}=50)$ (Fig. 1). The BMI $\left(19.9 \pm 3.2 \mathrm{~kg} / \mathrm{m}^{2}\right.$ vs. $\left.22.1 \pm 2.8 \mathrm{~kg} / \mathrm{m}^{2}, p=0.002\right)$ was significantly lower in the malnourished group. No significant differences were observed regarding the PSI score, PSI class, CCI score, or the metabolic index. No significant differences were observed regarding the rates for ICU admission, use of a vasopressor, and mechanical ventilation (Table 4).

The clinical outcomes following propensity score matching are presented in Fig. 2. No significant difference in short-term outcomes was observed according to the presence of malnutrition. The hospital length of stay and the hospital mortality rates were not different between the malnourished and well-nourished groups (hospital length of stay: $15.2 \pm 12.8$ days vs. $13.5 \pm 9.3$ days, $p=0.474$; hospital mortality: $5.4 \%$ vs. $2 \%, p=0.389$ ). However, hospital discharge status was significantly different between the groups. In the malnourished group, 70.3\% of patients were discharged to home, which was lower than the rate in the well-nourished group $(98 \%, p<$ 0.001 ), and $29.7 \%$ were transferred to long-term care facilities, which was higher than the rate in the well-nourished group $(2 \%, p<0.001)$. Moreover, long-term survival was also significantly lower in the malnourished group than in the well-nourished group (1-year survival: $67.6 \%$ vs. $90.0 \%, p=0.009$; 2-year survival: $59.5 \%$ vs. $86 \%$, $p=0.005)$ (Fig. 2). 
Table 4. The comparison according to baseline nutritional status in the elderly group

\begin{tabular}{|c|c|c|c|c|c|c|}
\hline \multirow[b]{2}{*}{ Variable } & \multicolumn{3}{|c|}{ Before propensity score matching } & \multicolumn{3}{|c|}{ After propensity score matching } \\
\hline & $\begin{array}{l}\text { Malnourished } \\
(\mathrm{n}=70)\end{array}$ & $\begin{array}{l}\text { Well-nourished } \\
\qquad(\mathrm{n}=61)\end{array}$ & $p$ value & $\begin{array}{l}\text { Malnourished } \\
(\mathrm{n}=37)\end{array}$ & $\begin{array}{l}\text { Well-nourished } \\
\qquad(\mathrm{n}=50)\end{array}$ & $p$ value \\
\hline Age & $77.2 \pm 6.6$ & $73 \cdot 5 \pm 5.8$ & 0.001 & $74.2 \pm 4.8$ & $74.6 \pm 5 \cdot 5$ & 0.698 \\
\hline Male sex & $40(57.1)$ & $34(55 \cdot 7)$ & 0.871 & $24(64 \cdot 9)$ & $26(52)$ & 0.230 \\
\hline $\mathrm{BMI}, \mathrm{kg} / \mathrm{m}^{2}$ & $19.8 \pm 3.0$ & $22.5 \pm 3.5$ & $<0.001$ & $19.9 \pm 3.2$ & $22.1 \pm 2.8$ & 0.002 \\
\hline CCI & $5.8 \pm 2.1$ & $5 \cdot 5 \pm 2.1$ & 0.387 & $5.6 \pm 2.0$ & $5.6 \pm 2.1$ & 0.915 \\
\hline PSI score & $84 \cdot 4 \pm 33 \cdot 3$ & $80.7 \pm 33.1$ & 0.524 & $83.1 \pm 34.6$ & $82.5 \pm 32.0$ & 0.928 \\
\hline PSI class & & & 0.687 & & & 0.816 \\
\hline I, II & $25(35 \cdot 7)$ & $28(45 \cdot 9)$ & & $14(37.8)$ & $21(42)$ & \\
\hline III & $15(21.4)$ & $12(19.7)$ & & $7(18.9)$ & $12(24)$ & \\
\hline IV & $23(32.9)$ & $16(26.2)$ & & $13(35.1)$ & $13(26)$ & \\
\hline $\mathrm{V}$ & $7(10)$ & $5(8.2)$ & & $3(8.1)$ & $4(8)$ & \\
\hline ICU admission & $10(14 \cdot 3)$ & $1(1.6)$ & 0.009 & $1(2.7)$ & o & 0.242 \\
\hline Use of vasopressor & $8(11.4)$ & $1(1.6)$ & 0.027 & $1(2.7)$ & 0 & 0.242 \\
\hline Mechanical ventilation & $9(12.9)$ & $1(1.6)$ & 0.016 & $1(2.7)$ & 0 & 0.242 \\
\hline \multicolumn{7}{|l|}{ Clinical outcome } \\
\hline Hospital stay, day & $17.6 \pm 19.7$ & $14.4 \pm 10.0$ & 0.246 & $15.2 \pm 12.8$ & $13 \cdot 5 \pm 9 \cdot 3$ & 0.474 \\
\hline Survival to discharge & $65(92.9)$ & $59(96.7)$ & 0.327 & $35(94.6)$ & $49(98)$ & 0.389 \\
\hline Discharge to home & $48(68.6)$ & $59(96.7)$ & $<0.001$ & $26(70.3)$ & $49(98)$ & $<0.001$ \\
\hline 1-yr survival & $44(62.9)$ & $55(90.2)$ & $<0.001$ & $25(67.6)$ & $45(90)$ & 0.009 \\
\hline 2-yr survival & $38(54.3)$ & $50(82.0)$ & 0.001 & $22(59 \cdot 5)$ & $43(86)$ & 0.005 \\
\hline
\end{tabular}

Values are presented as mean $\pm \mathrm{SD}$ or number (\%).

BMI, body mass index; CCI, Charlson comorbidity index; PSI, pneumonia severity index; ICU, intensive care unit.

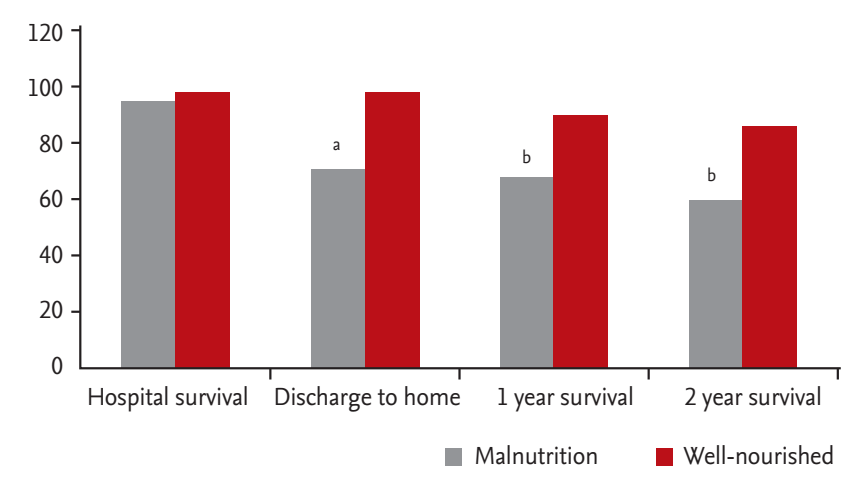

Figure 2. Difference of outcomes according to malnutrition in the elderly group after propensity score matching. The long-term survival was significantly lower in the malnourished group than in the well-nourished group (1-year survival: $67.6 \%$ vs. $90.0 \%, p=0.009 ; 2$-year survival: $59.5 \%$ vs. $86.0 \%, p=0.005) .{ }^{a} p<0.001,{ }^{b} p<0.01$.
Risk factors for 2-year mortality in the elderly group Age, nutritional status, PSI, and CCI were evaluated to identify independent risk factors for 2-year mortality in the elderly (Table 3). Univariate analyses showed that age (OR, 1.05; 95\% CI, 1.00 to $1.10 ; p=0.033$ ), malnutrition (OR, 4.07; 95\% CI, 1.94 to 8.51; $p<0.001$ ), CCI score (OR, 1.33; 95\% CI, 1.17 to 1.51; $p<0.001$ ), renal replacement therapy (OR, 3.23; 95\% CI, 1.26 to 8.30; $p=0.015$ ), and BMI (OR, 0.91; 95\% CI, 0.82 to $1.00 ; p=0.049$ ) were associated with 2-year mortality in the elderly. All of these variables were included in the Cox regression analysis model. Multivariate analyses showed that malnutrition (OR, 3.06; $95 \%$ CI, 1.44 to 6.50; $p=0.004)$, age (OR, 1.06; $95 \%$ $\mathrm{CI}, 1.01$ to $1.12 ; p=0.030$ ), and CCI score (OR, 1.37; 95\% CI, 1.18 to 1.58 ; $p<0.001$ ) were associated with 2-year mortality in the elderly. 


\section{DISCUSSION}

This study focused on the correlation between nutritional status at admission and long-term clinical outcomes in patients with CAP. Overall, $39.4 \%$ of the hospitalized patients with CAP were malnourished at admission. The proportion of malnourished patients was significantly higher $(53.4 \%$ vs. $11.9 \%, p<0.001)$ in the elderly group than in the non-elderly group. Approximately one-half of the elderly Korean patients with CAP were initially malnourished. Malnutrition in the elderly with CAP was associated with a worse patient condition at discharge and poor long-term mortality. Moreover, malnutrition was an independent predictor of 2-year mortality after discharge in the elderly patients. Considering the high prevalence of malnutrition and prognostic impact on long-term mortality in this group, a routine nutritional assessment at admission is mandatory as a first step for appropriate nutritional therapy.

Pneumonia is a common and serious problem in the elderly, and is associated with increased morbidity and mortality $[1,18]$. In this study, $66.2 \%$ of the patients who had been admitted to the hospital for CAP treatment were $>65$ years of age. To date, PSI or the "confusion, urea nitrogen, respiratory rate, blood pressure" (CURB)65 measurement are the most common tools used to stratify the severity of patients with CAP. Among parameters in both scoring systems, old age is the most significant. However, the effects of age itself on prognosis might be weaker exclusively among the elderly population [19]. In addition, PSI and the CURB-65 have been validated to assess short-term mortality in CAP patients but not long-term mortality. In this study, the PSI score was significantly lower in the elderly group than in the non-elderly group, which indicates that the elderly may have a higher rate of hospitalization than the non-elderly despite their relatively low disease severity. This difference in disease severity may be why there were no significant differences in the short-term outcomes, such as hospital mortality or length of hospital stay, between the elderly and non-elderly groups. Nevertheless, more patients in the elderly group were transferred to a nursing care facility at discharge and died during the 2-year follow-up period. It is not certain if this was related to the effects of aging itself or to CAP sequelae. This study suggests the possibility that poor long-term outcome

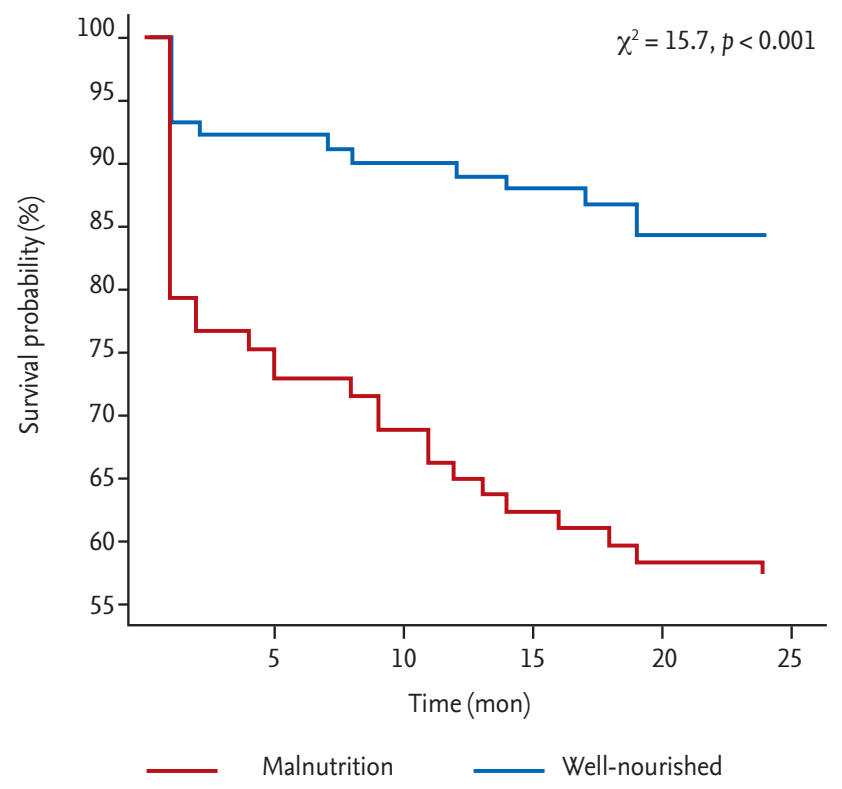

Figure 3. Kaplan-Meier survival curves and log-rank test. The probability of 2-year survival was decreased in elderly with malnutrition $\left(\chi^{2}=15.7, p<0.001\right)$.

is closely related to both malnutrition and comorbidities in the elderly group. These two factors were independently associated with 2-year mortality after discharge (hazard ratio [HR] for malnutrition, 3.06; 95\% CI, 1.44 to $6.50 ; p=0.004$ ) (HR for comorbidity, 1.37; 95\% CI, 1.18 to $1.58 ; p<0.001$ ), in line with previous data. In particular, malnutrition is a major contributor to increased morbidity and mortality, decreased function and quality of life, increased frequency and length of hospital stay, and higher healthcare costs $[5,6]$.

Globally, the over 65-year age group is a nutritionally vulnerable group, and the prevalence of malnutrition is as high as $40 \%$ in those admitted to the hospital [20]. In this study, $53.4 \%$ of the elderly with CAP were initially malnourished. The malnourished patients in the elderly group showed a lower rate of discharge to home and a lower long-term survival rate after discharge (Fig. 3), which suggests that elderly patients with CAP are nutritionally at risk and susceptible to morbidities and poor long-term outcomes. In data from a previous study, adverse consequences of malnutrition on physical and psychosocial outcomes were independent of underlying disease and disability [21]. Despite the resolution of pneumonia, malnourished elderly people have an increased risk of developing impaired muscle and re- 
spiratory function, which may lead to adverse long-term outcomes. Considering the increasing population of the elderly, it is worthwhile to evaluate the characteristics of this CAP patient subset.

Several studies have recognized the prognostic importance of malnutrition in elderly patients [22-24]. However, most studies are limited in that the definition or indicators of malnutrition have been limited to a single parameter [22-24]. To date, no single parameter is definitive for determining malnutrition in the elderly [25]. This study was limited due to the relatively small number of patients from a single institution and the retrospective design. First, clinical outcomes such as 1- and 2-year mortality rates were collected at each time point by confirming loss of the health insurance qualification; therefore, this study may have overestimated the mortality rate of all patients. Second, the index of frailty or sarcopenia of the patients at discharge was not collected, but was replaced by clinical results, such as the rate of discharge to home or nursing facility and longterm survival. Therefore, the cause-effect relationships between malnutrition and a poor long-term prognosis are unclear in this study. Additional studies are needed to confirm the process of malnutrition, frailty, and the long-term prognosis. Finally, there is also risk of bias when analyzing the risk of long-term mortality for a relatively small number of patients. Nevertheless, the purpose of this study was not to demonstrate its relevance but to provide a basis for a future large-scale population analyses. The main advantage of our study was that the diagnosis of malnutrition was routinely assessed and documented on admission by two clinical nutritionists, according to Academy of Nutritional and Dietetics consensus criteria, which are currently being used by many practitioners as part of the nutritional care process $[13,14]$.

In conclusion, malnutrition was associated with an increase in adverse discharge status and increased longterm mortality risk in the elderly with CAP. Nutritional status is a principal determinant of the health trajectory and outcome following discharge from the hospital in the elderly. Clinicians need to recognize that nutritional screening is important as an integral part of clinical practice, and a standardized format is needed to identify malnutrition and ultimately its treatment [26].

\section{KEY MESSAGE}

1. About $39 \%$ of the hospitalized patients with community-acquired pneumonia were malnourished at admission.

2. Malnutrition was associated with poor longterm outcomes in patients with community-acquired pneumonia, particularly the elderly.

\section{Conflict of interest}

No potential conflict of interest relevant to this article was reported.

\section{Acknowledgments}

This study was supported by the Research Institute for Convergence of Biomedical Science and Technology Grant (30-2016-005), Pusan National University Yangsan Hospital.

\section{REFERENCES}

1. Almirall J, Serra-Prat M, Bolibar I, Balasso V. Risk factors for community-acquired pneumonia in adults: a systematic review of observational studies. Respiration 2017;94:299-311.

2. Loeb M, High K. The effect of malnutrition on risk and outcome of community-acquired pneumonia. Respir Care Clin N Am 2005;11:99-108.

3. Tokgoz Akyil F, Yalcinsoy M, Hazar A, et al. Prognosis of hospitalized patients with community-acquired pneumonia. Pulmonology 2018;24:164-169.

4. Rodriguez-Pecci MS, Carlson D, Montero-Tinnirello J, Parodi RL, Montero A, Greca AA. Nutritional status and mortality in community acquired pneumonia. Medicina (B Aires) 2010;70:120-126.

5. Barker LA, Gout BS, Crowe TC. Hospital malnutrition: prevalence, identification and impact on patients and the healthcare system. Int J Environ Res Public Health 2011;8:514-527.

6. Jensen GL. Inflammation as the key interface of the medical and nutrition universes: a provocative examination of the future of clinical nutrition and medicine. JPEN J Parenter Enteral Nutr 2006;30:453-463.

7. Evans C. Malnutrition in the elderly: a multifactorial fail- 
ure to thrive. Perm J 2005;9:38-41.

8. Statics Korea. 2017 Statistics on the aged [Internet]. Daejeon (KR): Statics Korea, 2017 [cited 2018 Dec 6]. Available from: http://kostat.go.kr/portal/eng/pressReleases/11/1/ index.board?bmode $=$ read \&bSeq=\&aSeq=363974\&pageNo $=1$ \&rowNum $=10$ \&navCount $=10 \&$ currPg=\&sTarget=title\&sTxt=.

9. Lee JY, Yoo CG, Kim HJ, Jung KS, Yoo KH. Disease burden of pneumonia in Korean adults aged over 50 years stratified by age and underlying diseases. Korean J Intern Med 2014;29:764-773.

10. Yoo KH, Yoo CG, Kim SK, et al. Economic burden and epidemiology of pneumonia in Korean adults aged over 50 years. J Korean Med Sci 2013;28:888-895.

11. Kim HI, Kim SW, Chang HH, et al. Mortality of community-acquired pneumonia in Korea: assessed with the pneumonia severity index and the CURB-65 score. J Korean Med Sci 2013;28:1276-1282.

12. Chang HH. Community-acquired pneumonia in elderly patients. Korean J Med 2010;79:346-355.

13. White JV, Guenter P, Jensen G, et al. Consensus statement: Academy of Nutrition and Dietetics and American Society for Parenteral and Enteral Nutrition. Characteristics recommended for the identification and documentation of adult malnutrition (undernutrition). JPEN J Parenter Enteral Nutr 2012;36:275-283.

14. White JV, Guenter P, Jensen G, et al. Consensus statement of the Academy of Nutrition and Dietetics/American Society for Parenteral and Enteral Nutrition: characteristics recommended for the identification and documentation of adult malnutrition (undernutrition). J Acad Nutr Diet 2012;112:730-738.

15. Ho DE, Imai K, King G, Stuart EA. Matching as nonparametric preprocessing for reducing model dependence in parametric causal inference. Polit Anal 2007;15:199-236.

16. Ho DE, Imai K, King G, Stuart EA. MatchIt: nonparamet- ric preprocessing for parametric causal inference. J Stat Softw 2011;42:v042.io8.

17. Diamond A, Sekhon JS. Genetic matching for estimating causal effects: a general multivariate matching method for achieving balance in observational studies. Rev Econ Stat. 2013;95:932-945.

18. Stupka JE, Mortensen EM, Anzueto A, Restrepo MI. Community-acquired pneumonia in elderly patients. Aging health 2009;5:763-774.

19. Zhang ZX, Yong Y, Tan WC, Shen L, Ng HS, Fong KY. Prognostic factors for mortality due to pneumonia among adults from different age groups in Singapore and mortality predictions based on PSI and CURB-65. Singapore Med J 2018;59:190-198.

20. Kopelman P, Lennard-Jones J. Nutrition and patients: a doctor's responsibility. Clin Med (Lond) 2002;2:391-394.

21. Muhlethaler R, Stuck AE, Minder CE, Frey BM. The prognostic significance of protein-energy malnutrition in geriatric patients. Age Ageing 1995;24:193-197.

22. Ma HM, Tang WH, Woo J. Predictors of in-hospital mortality of older patients admitted for community-acquired pneumonia. Age Ageing 2011;40:736-741.

23. Riquelme OR, Riquelme OM, Rioseco ZML, Gomez MV, Cardenas G, Torres C. Community-acquired pneumonia in the elderly: clinical and nutritional aspects. Rev Med Chil 2008;136:587-593.

24. Vecchiarino P, Bohannon RW, Ferullo J, Maljanian R. Short-term outcomes and their predictors for patients hospitalized with community-acquired pneumonia. Heart Lung 2004;33:301-307.

25. Harris D, Haboubi N. Malnutrition screening in the elderly population. J R Soc Med 2005;98:411-414.

26. Jo HJ, Shin DB, Koo BK, Ko ES, Yeo HJ, Cho WH. The impact of multidisciplinary nutritional team involvement on nutritional care and outcomes in a medical intensive care unit. Eur J Clin Nutr 2017;71:1360-1362. 